\title{
Sex Determination by PCR in Single Bovine Blastomeres Biopsied by Extrusion Method at the 8-cell Stage
}

\author{
Yuichi Kameyama1*, Norimasa Shimada1, Naoko Kimura², \\ Yoshiaki Itagaki3 and Yoshiro Ishijima1 \\ ${ }^{1}$ Department of Bioproduction, Faculty of Bioindustry, Tokyo University of Agriculture, Abashiri-shi, \\ Hokkaido 099-2493, Japan \\ ${ }^{2}$ Graduate School of Agricultural Science, Tohoku University, Sendai-shi, Miyagi 981-8555, Japan \\ ${ }^{3} R$ \& D Center, Nihon Nosan Kogyo Co. Ltd, Tsukuba-shi, Ibaraki 300-2615, Japan
}

\begin{abstract}
We attempted to determine the sex by polymerase chain reaction (PCR) in single bovine blastomeres at the 8-cell stage. PCR was performed on male-specific primers attached to a bovine embryonic sex determination kit, an XY Selector. Embryos at the 8-cell stage were isolated by the EDTA method, and one (1/8 embryo), two (2/8 embryo), and four (4/8 embryo) blastomeres were subjected to $P C R$. The detection rates for male-specific PCR product were 25.8, 25.8 and $45.2 \%$ for 1/8, 2/8 and 4/8 embryos, respectively. In some embryos, despite detection of the male-specific product in $4 / 8$ embryos, the male-specific product was not detected in $1 / 8,2 / 8$ or both $1 / 8$ and 2/8 embryos derived from the same embryo (3.2, 3.2 and $16.1 \%$, respectively). Collecting single blastomeres by the extrusion method affected neither the rate of development to blastocysts nor the number of cells in blastocysts. PCR was performed in 1/8 embryos collected by the extrusion method, and the male-specific PCR product was detected. Nevertheless, in $27.5 \%$ of embryos, despite detection of male-specific $P C R$ product in $7 / 8$ embryos, the male-specific product was not detected in the 1/8 embryo from the same embryo. These findings indicated that collecting single blastomeres at the 8-cell stage allows the selection of the male embryo by $P C R$, and also the extrusion method is useful for biopsy of embryo at the 8-cell stage. Key words: Sexing, PCR, Bovine, Embryo, Biopsy
\end{abstract}

As sex determination methods for early mammalian embryos, a method using male-specific antigen, a method using sex difference in the rate of development,

Received: July 30, 1999

Accepted: December 17, 1999

*To whom correspondence should be addressed. a method which quantifies $X$ chromosome-linked enzymes, a method for detecting the $Y$ chromosome, and a method that amplifies $Y$ chromosome-specific nucleotide sequences and analyzes the product have been investigated [1, 2]. Among these methods, the method using a male-specific antigen and the method using sex difference in the rate of development are not always accurate, and the overall sexing rates of the method for measuring enzymes linked to the $X$ chromosome and the method for detecting the $Y$ chromosome are low. Therefore, the method for detecting $Y$ chromosome-specific nucleotide sequences by amplifying the sequence by polymerase chain reaction (PCR method) is considered to be promising, and has been developed for cattle [3-7], pigs [8] and mice $[9,10]$. As an advantage of the PCR method, tests can be rapidly and accurately performed on only a few cells, and commercial use in cattle has begun, but with the current biopsy method, blastomeres are collected by excising mural trophectoderm cells with the zona pellucida at the blastocyst stage, and there is a risk of affecting the normal development of the fetus. In mice, the extrusion method has been established, in which blastomeres at the 4- or 8-cell stage are biopsied by extruding from a small slit made in the zona pellucida [11, 12]. Collecting samples for PCR by this method may reduce injury to the embryo.

Therefore, in this study, we collected single blastomeres at the 8-cell stage by the extrusion method, and attempted to determine embryonic sex by the PCR method and investigated the developmental potency of the remaining $7 / 8$ embryos. 


\section{Materials and Methods}

\section{Oocyte recovery, in vitro maturation and in vitro fertilization}

Embryos at the 8-cell stage and blastocysts were produced by in vitro maturation and fertilization (IVMIVF). Recovery of oocytes and IVM-IVF were performed following the method reported by Kameyama et al. [13]. Oocytes were cultured for maturation in TCM199 supplemented with $5 \%$ fetal calf serum (FCS) for $21 \mathrm{~h}$. IVF was performed in m-BO medium [13] with frozen semen for artificial insemination. Spermatozoa were preincubated for $2.5 \mathrm{~h}$ for capacitation. After IVM, oocytes were incubated with capacitated spermatozoa at a density of $10-15 \times 10^{6} \mathrm{sperms} / \mathrm{ml}$ for $5 \mathrm{~h}$. Presumptive zygotes were co-cultured with cumulus cells in TCM199 supplemented with $1 \%$ FCS. The medium was exchanged on days 3,5 and 7 (day $0=I V F$ ) after IVF. The 8-cell stage embryos on day 3 and the blastocysts on day 9 were used in the experiments.

\section{Preparation of samples for $P C R$}

Samples for PCR were prepared from one (1/8 embryos), two (2/8 embryos), four (4/8 embryos), seven (7/ 8 embryos) and eight ( $8 / 8$ embryos) blastomeres, and blastocysts. The zona pellucida of $7 / 8$ and $8 / 8$ embryos and blastocysts was removed with PBS (-) containing $0.5 \%$ pronase. All embryos were washed $3-5$ times with PBS (-) by means of siliconized pipettes, then transferred to $0.5 \mathrm{ml}$ microtubes containing $8 \mu \mathrm{l}$ of sterilized pure water. The prepared samples were stored at $-20^{\circ} \mathrm{C}$ until use for PCR.

The $1 / 8$ embryos were prepared by the EDTA method and the extrusion method, 2/8 and 4/8 embryos by the EDTA method, and 7/8 embryos by the extrusion method. In the EDTA method, zona pellucida-removed embryos at the 8-cell stage were pipetted in PBS (-) supplemented with $0.02 \%$ EDTA, and each $1 / 8,2 / 8$ and $4 / 8$ embryo was separated. In the extrusion method, $1 / 8$ embryos was extruded with a micromanipulator, and the remaining blastomeres were used as $7 / 8$ embryos. Micromanipulation was performed in $\mathrm{M} 2$ medium [14]. The outer and inner diameters of the holding pipettes were 180 and $25 \mu \mathrm{m}$, respectively. Those of injection pipettes were 20 and $10 \mu \mathrm{m}$, respectively, and the tip was beveled to $35^{\circ}$, and a spike was made by using a microforge. To extrude blastomeres, a part of the zona pellucida was incised, and M2 medium was blown into the embryo via the injection pipette that was in serted at another site.

\section{3. $P C R$}

PCR was performed following the instructions and using only male-specific primers attached to the bovine sex determination kit (XY Selector, Itoham Foods Inc.). An enzyme mixture solution was prepared at a ratio of $2 \times$ reaction solution: enzyme solution $=9.9 \mu \mathrm{l}: 0.1 \mu \mathrm{l}$, and 9.5-9.9 $\mu$ was added to a sample. As a PCR apparatus, QUICK THERMO PERSONAL (NIPPON GENETIC Co.) was used. The program consisted of $2 \mathrm{~min}$ initial at $95^{\circ} \mathrm{C}$ and 44 cycles of $30 \mathrm{sec}$ at $95^{\circ} \mathrm{C}, 10 \mathrm{sec}$ at $50^{\circ} \mathrm{C}$, and 10 sec at $70^{\circ} \mathrm{C}$. The PCR product was analyzed by mini-gel electrophoresis. To $15 \mu \mathrm{l}$ of the PCR product, $3 \mu \mathrm{l}$ of BPB solution (25\% Glycerol, 0.05\% Bromophenol Blue, $0.05 \%$ Xylene Cyanol) was added, then loaded onto a gel. Electrophoresis was performed with $3 \%$ agarose gel (NuSieve GTG Agarose, FMC Bioproducts) at $50 \mathrm{~V}$ for approximately $70 \mathrm{~min}$. After electrophoresis, the gel was stained with ethidium bromide for $50 \mathrm{~min}$, and the appearance of the male-specific PCR product was examined under ultraviolet illumination.

\section{Viability of $7 / 8$ embryo biopsied by the extrusion method}

Intact $8 / 8$ embryos, 8/8 embryos kept on a warm plate $\left(37^{\circ} \mathrm{C}\right)$ for a time required for micromanipulation (10 $\mathrm{min}$ ), 7/8 embryos prepared by destroying one blastomere, and 7/8 embryos from which one blastomere was removed by the extrusion method were co-cultured with cumulus cells for $144 \mathrm{~h}$ (until day 9), and the development of embryos to blastocysts was observed. Destruction of a single blastomere was performed according to the extrusion method. The blastocysts derived from intact $8 / 8$ embryos and $7 / 8$ embryos prepared by removing one blastomere by the extrusion method were stained by double fluorescence staining [13, 15], and the numbers of cells in the inner cell mass and the trophectoderm were counted after $120 \mathrm{~h}$ of culture (day 8).

\section{Results}

\section{Sex determination in $1 / 8,2 / 8$, and $4 / 8$ embryos prepared by the EDTA method}

The sex of the $1 / 8,2 / 8$, and $4 / 8$ embryos prepared by the EDTA method was determined (Table 1). As the control, 8/8 embryos and blastocysts were used. The detection rates for male-specific PCR product were 25.8, $25.8,45.2,40.0$ and $43.8 \%$ for $1 / 8,2 / 8,4 / 8,8 / 8$ embryos, and blastocysts, respectively. Sets of $1 / 8,2 / 8$, and $4 / 8$ embryos derived from the same embryo were individually prepared, but the male-specific PCR product detection rates of each embryo in a set were not 
consistent.

Consistency of sex determination among the $1 / 8,2 / 8$ and $4 / 8$ embryos is summarized in Table 2. The ratio of embryo sets in which male-specific PCR product was detected in all embryos was $22.6 \%$, and that of embryo sets in which no male-specific PCR product was detected was $54.8 \%$, so that the percentage of embryo sets that obtained consistent sex determination was $77.4 \%(24 / 31)$. In the embryo sets with inconsistent determinations, male-specific PCR product was detected in $4 / 8$ embryos, but not in $1 / 8(3.2 \%)$ or $2 / 8(3.2 \%)$, or both $1 / 8$ and $2 / 8$ embryo (16.1\%).

\section{Biopsy of embryos at the 8-cell stage by the extrusion method}

The efficiency of collecting blastomeres by the extrusion method is shown in Table 3. Of 116 embryos tested, one or two blastomeres were collected in $78.4 \%$

Table 1. Sex determination by PCR in $1 / 8,2 / 8$ and $4 / 8$ embryos produced by the EDTA method

\begin{tabular}{ccc}
\hline $\begin{array}{c}\text { Type of } \\
\text { embryo }\end{array}$ & $\begin{array}{c}\text { No. of embryos } \\
\text { applied }\end{array}$ & $\begin{array}{c}\text { No. and (\%) of embryos } \\
\text { detected male specific signals }\end{array}$ \\
\hline $1 / 8^{*}$ & 31 & $8(25.8)$ \\
$2 / 8^{*}$ & 31 & $8(25.8)$ \\
$4 / 8^{*}$ & 31 & $14(45.2)$ \\
$8 / 8$ & 10 & $4(40.0)$ \\
blastocyst & 16 & $7(43.8)$ \\
\hline
\end{tabular}

*Sets of $1 / 8,2 / 8$, and $4 / 8$ embryos derived from the same embryo were individually prepared. (a single blastomere extruded: $59.5 \%$, two blastomeres extruded: $19.0 \%$ ), but in $12.1 \%$ of the embryos, three or more blastomeres were extruded, and extruded blastomeres were destroyed in $9.5 \%$.

In the $7 / 8$ embryos obtained by removing one blastomere by the extrusion method, $60.9 \%$ developed to morulae, and $47.8 \%$ developed to blastocysts (Table 4). The ratio of development to blastocysts from the $7 / 8$ embryos did not significantly differ from those from the intact embryos at the 8 -cell stage $(62.8 \%)$, from the embryos at the 8-cell stage kept on a warm plate for the time required for micromanupilation $(50.0 \%)$, or from the $7 / 8$ embryos prepared by destroying one blastomere (45.0\%) (P>0.05).

The blastocysts derived from the $7 / 8$ embryos prepared by the extrusion method consisted of 63.1 cells on average (Table 5). Among these cells, the number of cells in the inner cell mass (ICM) was $14.3(22.7 \%)$.

Table 2. Consistency of sex determined by PCR in sets of $1 / 8,2 / 8$ and $4 / 8$ embryos produced by the EDTA method from the same embryo

\begin{tabular}{cccc}
\hline \multicolumn{2}{l}{ Detection of male specific signals in } & & \multirow{2}{*}{$\begin{array}{c}\text { No. and }(\%)^{*} \\
\text { of sets }\end{array}$} \\
\cline { 1 - 3 } $1 / 8$ & $2 / 8$ & $4 / 8$ & \\
\cline { 1 - 2 }$\sigma^{\nearrow}$ & $\sigma^{\nearrow}$ & $\sigma^{\nearrow}$ & $7(22.6)$ \\
$\sigma^{\nearrow}$ & - & $\sigma^{\nearrow}$ & $1(3.2)$ \\
- & $\sigma^{\nearrow}$ & $\sigma^{\nearrow}$ & $1(3.2)$ \\
- & - & $\sigma^{\nearrow}$ & $5(16.1)$ \\
- & - & - & $17(54.8)$ \\
\hline
\end{tabular}

*Percentages were calculated by the total number of sets.

Table 3. Result of manipulation by the extrusion method at the 8-cell stage

\begin{tabular}{ccccc}
\hline \multirow{2}{*}{$\begin{array}{c}\text { No. of } \\
\text { embryos } \\
\text { manipulated }\end{array}$} & \multicolumn{4}{c}{ No. and $(\%)^{*}$ of embryos classified by the result of manipulation as } \\
\cline { 2 - 5 } & $\begin{array}{c}\text { Extrusion of } \\
\text { one blastomere }\end{array}$ & $\begin{array}{c}\text { Extrusion of } \\
\text { two blastomeres }\end{array}$ & $\begin{array}{c}\text { Extrusion over } \\
\text { three blastomeres }\end{array}$ & $\begin{array}{c}\text { Breakdown of } \\
\text { blastomeres }\end{array}$ \\
\hline 116 & $69(59.5)$ & $22(19.0)$ & $14(12.1)$ & $11(9.5)$ \\
\hline
\end{tabular}

*Percentages were calculated by the number of embryos manipulated.

Table 4. Effect of manipulation on the development of biopsied embryos

\begin{tabular}{lccr}
\hline \multirow{2}{*}{ Treatment of embryo } & $\begin{array}{c}\text { No. of embryos } \\
\text { cultured }\end{array}$ & No. and (\%) of embryos developed to \\
\cline { 3 - 4 } & 43 & $32(74.4)$ & Blastocyst \\
\hline Control & 20 & $15(75.0)$ & $27(62.8)$ \\
Leaving for 10 minutes & 20 & $14(70.0)$ & $10(50.0)$ \\
Destroy of one blastomere & 23 & $14(60.9)$ & $9(45.0)$ \\
Biopsy of one blastomere & & & $11(47.8)$ \\
\hline
\end{tabular}

${ }^{*}$ On warm plate $\left(37^{\circ} \mathrm{C}\right)$. 
Table 5. Numbers of cells in blastocysts derived from $7 / 8$ embryos biopsied by the extrusion method

\begin{tabular}{ccccc}
\hline \multirow{2}{*}{$\begin{array}{c}\text { Origin of } \\
\text { blastocysts }\end{array}$} & $\begin{array}{c}\text { No. of } \\
\text { blastocysts }\end{array}$ & \multicolumn{3}{c}{ Numbers of cells* and $(\%)^{* *}$ of } \\
\cline { 3 - 5 } & 10 & $14.3 \pm 2.2(22.7)$ & $48.8 \pm 6.8(77.3)$ & $63.1 \pm 8.3(100)$ \\
$7 / 8$ & 12 & $16.7 \pm 2.5(24.1)$ & $52.4 \pm 5.8(75.9)$ & $69.1 \pm 7.3(100)$ \\
\hline $8 / 8$ & & &
\end{tabular}

${ }^{*}$ Mean \pm S.E.

${ }^{* *}$ Percentages were calculated by the total numbers of cells.

Table 6. Consistency of sex determined by PCR in $1 / 8$ and $7 / 8$ embryos produced by the extrusion method

\begin{tabular}{|c|c|c|c|}
\hline \multirow{2}{*}{$\begin{array}{l}\text { Detection of male } \\
\text { specific signals } \\
\text { in } 1 / 8 \text { embryos }\end{array}$} & \multicolumn{3}{|c|}{$\begin{array}{c}\text { No. and }(\%) \text { of sets classified by the detection of } \\
\text { male specific signals in } 7 / 8 \text { embryos }\end{array}$} \\
\hline & $\sigma^{7}$ & - & Total \\
\hline$\sigma^{\top}$ & $4(10.0)$ & $0(0.0)$ & $4(10.0)$ \\
\hline- & $11(27.5)$ & $25(62.5)$ & $36(90.0)$ \\
\hline Total & $15(37.5)$ & $25(62.5)$ & $40(100.0)$ \\
\hline
\end{tabular}

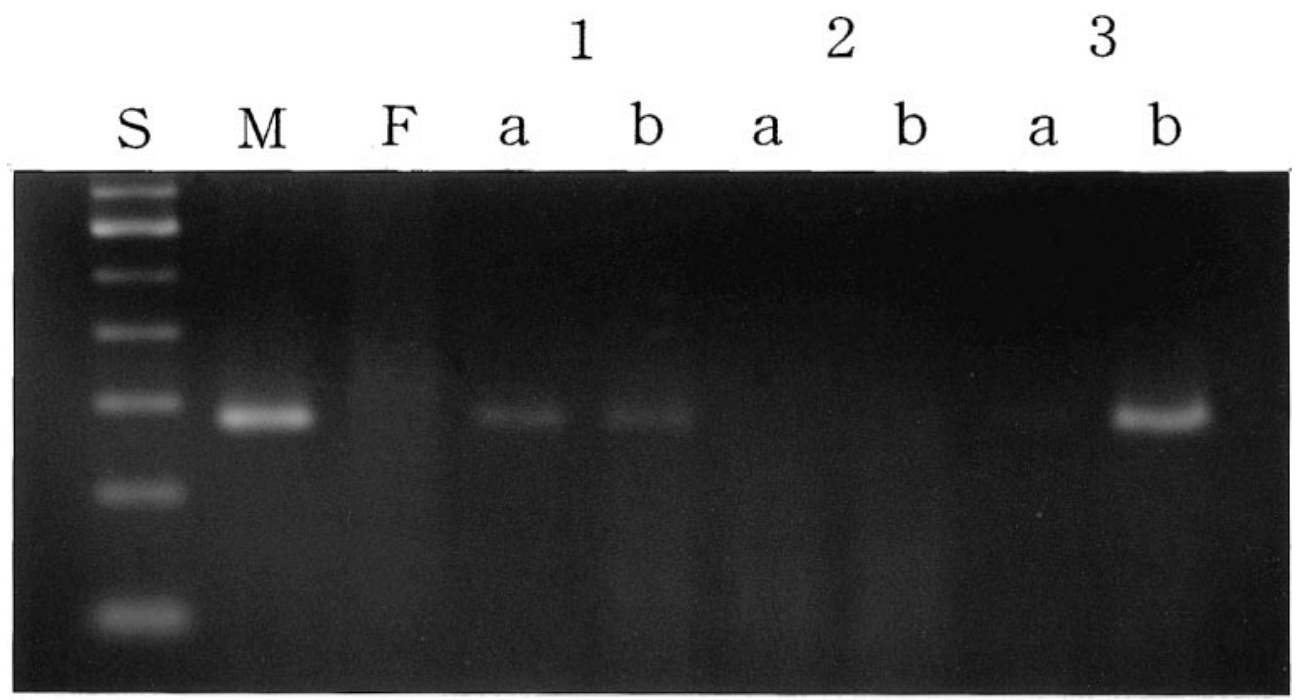

Fig. 1. Agarose gel electrophoresis of PCR products from $1 / 8$ (a) and $7 / 8$ (b) embryos produced by the extrusion method. Each set was derived from the same embryo. S:size marker, M: purified male DNA, F: purified female DNA. Male-specific PCR products (about $300 \mathrm{bp}$ ) were detected in lane $\mathrm{M}, 1 \mathrm{a}, 1 \mathrm{~b}$ and $3 \mathrm{~b}$.

These numbers of cells did not significantly differ from those of the blastocysts derived from the intact $8 / 8 \mathrm{em}$ bryos (total number of cells: 69.1, ICM cells: 16.7).

\section{Sex determination in $1 / 8$ and $7 / 8$ embryos prepared by the extrusion method}

The sex of $1 / 8$ and $7 / 8$ embryos prepared by the extrusion method was determined and the results were classified by consistency (Table 6). The male-specific PCR product detection rates were $10.0 \%$ of the $1 / 8 \mathrm{em}$ bryos, and $37.5 \%$ of the $7 / 8$ embryos, showing that the results were not consistent. The male-specific PCR product detection rate for the $1 / 8$ embryos significantly differed from the theoretical value of $50 \%(P<0.05)$. The embryo sets in which male-specific PCR product was detected in both $1 / 8$ and $7 / 8$ embryos was $10 \%$, and 
those in which male-specific PCR product was not detected in either $1 / 8$ or $7 / 8$ embryos was $62.5 \%$. Therefore, consistency in sex determination was obtained in $72.5 \%(29 / 40)$ of the embryo sets. In the sets for which consistency was not obtained, male-specific PCR product was detected in the $7 / 8$ embryos, but not in the $1 / 8$ embryos (27.5\%).

\section{Discussion}

In blastomeres separated by EDTA, we investigated the number of blastomeres required for sex determination of bovine embryos at the 8-cell stage. Kirkpatrick and Monson [5] collected 2-10 blastomeres from embryos at the stages from compacted morulae to expanding blastocysts, and determined the sex with male-specific and gender neutral primers. They reported that PCR product common to both sexes was detected in $95 \%$ of the samples, and the sex ratio (male ratio) was $65.8 \%$. Macháty et al. [6] collected single blastomeres from embryos at the 16- and 32-cell stages, and determined the sex with male-specific and gender neutral primers. The detection rate for PCR product common to both sexes was $95 \%$, but the sex ratio was not mentioned. In this study, only male-specific primers were used, so that samples in which male-specific PCR product was detected were regarded as male. In 4/8 embryos, $45.2 \%$ were male, which did not differ from the expected male ratio of $50 \%$, showing that four blastomeres are sufficient to obtain almost completely reliable sex determination of bovine embryos at the 8-cell stage. The male-specific PCR product could be detected in single blastomeres at the 8-cell stage, showing that male embryos can be selected by using a 1/8 embryo.

In the PCR method, Y chromosome-specific nucleotide sequences are amplified and used for sex determination, the success rate in selecting sex for birth should be $100 \%$. Nevertheless, because a success rate of less than $100 \%$ in cattle was mentioned in many reports $[3,5,7]$, it is difficult to obtain $100 \%$ in cattle, unlike mice, for which $100 \%$ was reported [9]. In this study, determined sex was inconsistent among 1/8, 2/8, and $4 / 8$ derived from the same embryo, and between $1 /$ 8 and 7/8 from the same embryo. Some male embryos may be regarded as female when determination is made with two or fewer blastomeres. As a reason why malespecific DNA was not amplified, sampling error, contamination during sampling (effects of FCS and BSA on enzyme reaction, contamination of DNA due to cell debris and spermatozoa adhering to the zona pellucida surface), and difference in heat conductance among models of PCR apparatus were considered [16]. Because $1 / 8$ and 2/8 embryos are small and easily adhere to the wall of a pipette, it is difficult to confidently put these embryos into PCR tubes. It is necessary to use siliconized pipettes and reduce the carry-in solution as much as possible.

On sex determination of the $1 / 8$ embryos collected by the EDTA method or the extrusion method, malespecific PCR product was detected in both samples. In the extrusion method, a small amount of culture medium was blown into the zona pellucida to extrude blastomeres from a incision, and it is not necessary to strain blastomeres and nuclei by aspiration, unlike in the aspiration method or enucleation method. Therefore, blastomeres collected by the extrusion method are less injured at the cell membrane and nuclei, indicating that this method is appropriate for gene diagnosis including sex determination of embryos. But the male-specific PCR product detection rate in 1/8 embryos obtained by the extrusion method was lower than that in 1/8 embryos obtained by the EDTA method, suggesting that some blastomeres were injured during extrusion from the slit made in the zona pellucida. Injury to blastomeres can be prevented by making a larger slit in the zona pellucida, but the biopsied embryos for which hatching was made easier may be likely to be injured during embryo transfer. In this study, micromanipulation was performed in conventional M2 medium. Addition of sucrose and EDTA may cause constriction of blastomeres, and make separation easier, which may make the extrusion method easier and more reliable. Takeuchi et al. [17] reported that they collected human single blastomeres at the 4- and 8-cell stages by the enucleation, aspiration, or extrusion method, and the viability of embryos at the 4-cell stage was not affected by the aspiration or extrusion method, and the viability of embryos at the 8-cell stage was not affected by either method. In cattle, single blastomeres at the 16- to 32-cell stages collected by the aspiration method were transplanted, and fertility similar to that of the control subjects was obtained [6]. In this study, the 7/8 embryos obtained by the extrusion method developed to blastocysts without difference from the development of intact embryos at the 8-cell stage, showing that the collection of embryos by the extrusion method causes less inhibition of embryonic development.

The above findings showed that male embryos can be selected by the PCR method with a single blastomere at the 8-cell stage obtained by the extrusion method, and the extrusion method results in less injury to embryos. It is therefore useful for biopsy. 


\section{Acknowledgments}

We are grateful to associated members at the Doto factory of Nippon Food Packer Inc., and the Hokkaido Higashimokoto Meat Inspection Center for cooperation in ovarian sampling. And we thank Itoham Inc. Central Research Institute for providing male-specific primers attached to the bovine embryonic sex determination kit, the XY Selector.

\section{References}

1) J afar, S.I. and Flint, A.P.F . (1996): Sex selection in mammals: A review. Theriogenology, 46, 191-200.

2) Reubinoff, B.E. and Schenker, J.G. (1996): New advances in sex preselection. Fertil. Steril., 66, 343350.

3) Herr, C.M., Holt, N.A., Matthaei, K.I . and Reed, K.C. (1990): Sex of progeny from bovine embryos sexed with a rapid $\mathrm{Y}$-chromosome-detection assay. Theriogenology, 33, 247.

4) Itagaki, Y., Sato, S., Shitanaka, Y., Kudo, T., Yamaguchi, Y. and Sutou, S. (1993): Sexing of bovine embryos with male-specific repetitive DNA by polymerase chain reaction: Sexing of bovine embryos and production of calves with predicted sex. J. Reprod. Dev., 39, 65-72.

5) Kirkpatrick, B.W. and Monson, R.L. (1993): Sensitive sex determination assay applicable to bovine embryos derived from IVM and IVF. J . Reprod. Fert., 98, 335340.

6) Macháty, Z., Páldi, A., Csáki, T., Varga, Z., Kiss, I., Bárándi, Z. and Vajta, G. (1993): Biopsy and sex determination by PCR of IVF bovine embryos. J . Reprod. Fert., 98, 467-470.

7) Watanabe, S., Takahashi, S., Konishi, H., Imai, H., Awata, T., Takahashi, H., Masuda, H. and Yasue, H. (1992): An attempt of embryo sexing using polymerase chain reaction in cattle. Anim. Sci. Technol. (J pn.), 63, 715-720.

8) Fajfar-Whetstone, C.J ., Rayburn, A.L., Schook, L.B. and Wheeler, M.B. (1993): Sex determination of porcine preimplantation embryos via $Y$-chromosome specific DNA sequences. Anim. Biotechnol., 4, 183-193.

9) Bradbury, M.W., I sola, L.M. and Gordon, J.W. (1990): Enzymatic amplification of a $Y$ chromosome repeat in a single blastomere allows identification of the sex of preimplantation mouse embryos. Proc. Natl. Acad. Sci. USA, 87, 4053-4057.

10) Mulder, L.C., Sacco, M.G., Mangiarini, L., Brown, J ., Collotta, A., Villa, A., De Giovanni, A.M., Vezzoni, P. and Clerici, L. (1993): Preimplantation embryo sexing by polymerase chain reaction amplification of the sry gene on single mouse blastomeres. Genet. Anal. Tech. Appl., 10, 147-149.

11) Sueoka, K., Hashiba, T., Asada, H., Kuroshima, M., Kuji, N., Kobayashi, T., Yoshimura, Y. and Nozawa, S. (1996): The genetic diagnosis of gametes and early embryo. J . Fert. Implant. Tokyo, 13, 20-26.

12) Tarín, J .J . and Handyside, A.H. (1993): Embryo biopsy strategies for preimplantation diagnosis. Fertil. Steril., 59, 943-952.

13) Kameyama, Y., Tanno, N. and I shijima, Y. (1996): Cell number of inner cell mass of bovine blastocyst fertilized and cultured in vitro. Anim. Sci. Agric. Hokkaido, 38, 39-42.

14) Quinn, P., Barros, C. and Whittingham, D.G. (1982): Preservation of hamster oocytes to assay the fertilizing capacity of human spermatozoa. J . Reprod. Fert., 66, 161-168.

15) I wasaki, S., Yoshiba, N., U shijima, H., Watanabe, S. and Nakahara, T. (1990): Morphology and proportion of inner cell mass of blastocysts fertilized in vitro and in vivo. J . Reprod. Fert., 90, 279-284.

16) Takai, N., Watanabe, S. and Masuda, H. (1995): Application of urasil $\mathrm{N}$-glycosylase treatment for bovine embryo sexing using polymerase chain reaction. Anim. Sci. Technol. (J pn.), 66, 383-385.

17) Takeuchi, K., Kaufmann, R.A., Sandow, B.A., Beebe, S.J ., Morsy, M. and Hodgen, G.D. (1992): Preclinical models for human pre-embryo biopsy and genetic diagnosis. I. Efficiency and normalcy of mouse preembryo development after different biopsy techniques. Fertil. Steril., 57, 425-430. 